\title{
Integrating Variational Level Set Method and Fuzzy c- Means to Automatically Segment the MRI Brain Images
}

\author{
Pratibha Singh \\ Department of C.S.E \\ GBPEC, Pauri Garhwal \\ Uttarakhand, India
}

\author{
H.S. Bhadauria, Ph.D \\ Department of C.S.E \\ GBPEC, Pauri Garhwal \\ Uttarakhand, India
}

\author{
Annapurna Singh, Ph.D \\ Department of C.S.E \\ GBPEC, Pauri Garhwal \\ Uttarakhand, India
}

\begin{abstract}
The main aim of this paper is to provide a method that could easily delineate the exact location of tumor region from brain MRI images by integrating the fuzzy c-means and level set method. The proposed method smoothly exploits the spatial function during FCM clustering which in turn proves the automaticity of the method by dividing the original image into clusters and then using one cluster for automatic initialization. Since automating the process reduces the time utilization of processing thereby making the work less tedious, hence if considered it could be competent tool in future. Another problem associated when utilizing manual methods is that it may lead to different results when produced by different medical experts which can be completely erased while using this method. Secondly, the proposed method uses the level set method to find the contour of tumor region in the original image which helps in situations where image changes their topologies by merging or splitting in two. Thus, the proposed method is using the convenient variational level set method in place of traditional level set method thereby eliminating one more drawback of re-initializing the contour several times during image segmentation.
\end{abstract}

\section{Keywords}

Image segmentation, level set methods, Fuzzy c-means, defuzzification, variational level sets.

\section{INTRODUCTION}

Image segmentation is the technique of distributing any digital image into one or more region of interest (ROI). In this paper the segmentation is considered in terms of medical imaging. The proposed algorithm can be significantly used in general segmentations as well. The segmented images in the field of medical imaging are used to examine the anatomical structures and also in diagnosis and assisting in surgical planning

There are several neurological circumstances that amend the volume, shape and distribution of brain tissue ,therefore, MRI is the imaging modality favored[1].

Fuzzy set theory has become progressively enticing amidst several image segmentation techniques available due to its robustness for uncertainty and can possess information with much more accuracy and simplicity than other techniques of segmentation. Since FCM after its introduction in 1965 by LotfiZadeh has some weaknesses and several attempts[2] like using the objective function FCM, using the neighbor pixel in addition to the pixel and even pixel division has been done. FCM clustering is well known as an unsupervised technique for cluster analysis in which the data points are allocated to clusters in a fuzzy sense, as in fuzzy logic, rather than belonging to one cluster.

The primitive active contour model designed by Kass et al. [3] extract the objects from an image by moving the explicit parametric curve yet it hold on to some drawbacks such as trouble in handling topological changes[4] and its need of parameterization. The fundamental objective is to represent the contours as the zero level set of an implicit function defined in a higher dimension, commonly cited as level set function which further is emerged according to partial differential equation (PDE) as categorized by level set method (LSM). Thus, the approach entertains a variety of advantages such as automatically handling the topological changes[9] and allows the level set function to retain its function on a fixed grid. Earlier this PDE needs to be converted to an evolution PDE of parametrized curve using Eulerian formulation, hence, an alternative is adopted. By minimizing an energy function defined on the level set function, the evolution PDE can be derived more conveniently and are usually[10] referred as variational level set methods.

The objective of this research is to suggest a new technique for MRI brain segmentation using integrated level set method and fuzzy c-means (FCM). Fuzzy c-means is been applied so that the procedure can process the segmentation of images automatically. The main advantage of automating the process is that it has made the processing much less tedious and not at all time consuming.

\section{RELATED WORK}

\subsection{Variational Level Set Method}

While executing the traditional level set methods it is an indispensable requirement to keep the evolving level set function contiguous to the signed distance function[6] to prevent it to be too steep or flat near the surface. The broadly used numerical strategy to maintain the interface evolution stable and to ensure usable results, a process known as reinitialization is utilized. Nevertheless, re-initializing produces several conflicts between the theory and implementation of level sets as it leads to some abominable effects such as shifting the zero level set away from its original location. The problem of how and when to apply re-initialization has now become an issue.

There are several impressive ideas by which we can implement re-initialization. Fast marching method proposed by Sethian[9] readily calculates the signed distance function by solving the Eikonal equation on both sides of the interface. Fast sweeping method[5] is another convenient method for solving this equation. 
However, a new variational formulation proposed by Chunming Li[7],that pushes the level set function to be close to signed distance function thereby completely eliminating the costly re-initialization process is usually practiced nowadays. The variational energy functional encompasses an internal energy term and an external energy term, subsequently. The internal energy term condemns the deviation of the level set function from a signed distance function, while the external energy term drives the motion of the zero level set to the desired image features. The gradient flow that minimizes the overall energy functional is the resulting evolution of the level set function is commonly and automatically kept as a close signed distance function during the evolution due to the internal energy.

\subsubsection{Varitional level set formulation without re- initialization considering penalizing energy}

As discussed by Chunming $\mathrm{Li}$, we can formulate the general interface evolution problem considering the constraint on signed distance i.e. Eikonal equation, as the given constrained minimization problem,

$$
\min _{\phi} F(\phi) \text { subject to }|\nabla \phi|=1
$$

Thus, to avoid re-initialization a penalization term is introduced as shown below,

$$
\mathrm{P}(\phi)=\lambda \int_{\Omega} 1 / 2(|\nabla \phi|-1)^{2} \mathrm{dx} d \mathrm{y}
$$

The following integral will help in characterizing how close a function $\phi$ is to the signed distance function in $\Omega \subset \mathrm{R}^{2}$ Using $\mathrm{P}(\phi)$, we introduce the following variational formulation,

$$
\varepsilon(\phi)=\mu \mathrm{P}(\phi)+\varepsilon_{\mathrm{m}}(\phi)
$$

where $\mu$ is the parameter controlling the effect of penalizing the deviation of $\phi$ from the signed distance and $\varepsilon_{\mathrm{m}}(\phi)$ is the energy that would drive the motion of zero level curve of $\phi$. In this paper, the gradient flow that will minimize the functional $\varepsilon$ is given below, also known as Gateaux derivative[7],

$$
\frac{\partial \phi}{\partial t}=-\frac{\partial \varepsilon}{\partial \phi}
$$

Since $\varepsilon_{\mathrm{m}}$ depends on image data thus, referred as external energy while $\mathrm{P}(\phi)$ wholly depends on $\phi$ therefore, known as internal energy.

\subsection{Fuzzy c-means clustering}

Fuzzy sets are usually referred as expansions of classical sets. Unlike classical sets, fuzzy sets permits partial membership. An arbitrary element $\mathrm{p}$ can belong to different fuzzy sets with different degrees of membership. This provides a much more reliable approach to solve problems intrinsically affected by uncertainty. Firstly[11], the FCM algorithm authorize pixels to each category by using fuzzy memberships. Let us assume $\mathrm{P}=(\mathrm{p} 1, \mathrm{p} 2, ., \mathrm{pN})$ denotes an image with $\mathrm{N}$ pixels to be partitioned into c clusters, where $p_{i}$ represents multispectral data. The algorithm [8]discussed is an iterative escalation that minimizes the cost function defined as follows:

$$
\mathrm{J}=\sum_{j=1}^{N} \sum_{i=1}^{c} u_{i j}^{m}\left\|p_{j}-v_{i}\right\|^{2}
$$

where $u_{i j}$ means the membership of pixel $p_{j}$ in the $i^{\text {th }}$ cluster, $v_{i}$ is the ith cluster center, $\|$.$\| is a norm metric, and m$ is a constant. The parameter $\mathrm{m}$ controls the fuzziness of the resulting partition.
Since in an image the neighborhood pixels are highly correlated i.e. they share similar feature values and probability to belong to the same cluster which is an important characteristics in spatial clustering. This spatial relationship can help in improving segmentation results. To escapade the spatial data, a spatial function is defined and is shown below,

$$
h_{i j}=\sum_{k \epsilon N B\left(p_{j}\right)} u i k
$$

where $\mathrm{NB}\left(\mathrm{p}_{\mathrm{j}}\right)$ represents a square window centered on pixel $\mathrm{p}_{\mathrm{j}}$ in the spatial domain. The spatial functions consolidate the original membership thereby maintaining unchanged clustering result in a homogenous region and for a noisy pixel, the function weakens weighting of noisy cluster by labels of its neighboring pixels which is a rectification of misclassification of pixels from noisy regions. Then, each pixel is assigned the specific cluster according to its maximal membership and is known as defuzzification[1].

\section{PROPOSED METHOD}

In this paper, we are going to propose an integrated method which combines fuzzy c-means and level set method without re-initialization. The main steps involved in the proposed method involves the integration of fuzzy c-means and level set method algorithm can be explained as follows:

1. Read the input image.

2. Apply the fuzzy c-means to divide into clusters.

3. Choose the fuzzy cluster to define initial contour for modified level set method.

4. Use the above contour in input image in level set method to obtain the desired image segmentation.

5. Display the segmented image.

The flow chart of proposed algorithm can be shown as follows:

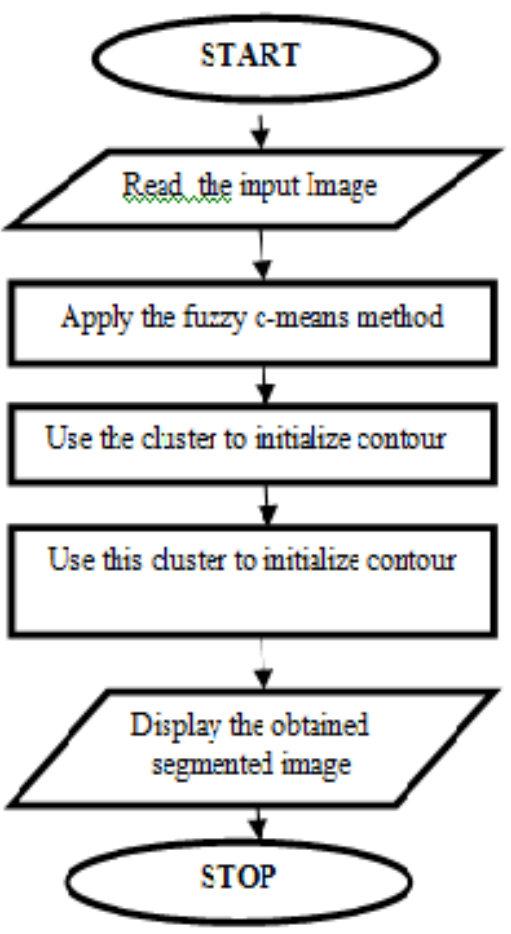

Fig 1: Proposed algorithm 


\section{CONCLUSIONS AND FUTURE WORK}

The above discussed algorithm when processed will definitely show the utility of our proposed method in segmenting the MRI image particularly with weak object boundaries, which is very difficult for fuzzy c-means and level set method to apply. In this paper, our proposed algorithm completely eliminates the need of manually initializing the contour for level set method. Our proposed method demonstrate relatively good performance for objects with weak boundaries specially when dealing with MRI images. Since the method proposed is automatic therefore it is quick and relatively free from errors as well.

\section{REFERENCES}

[1] Keh-Shih Chuang, Hong-Long Tzeng, Sharon Chen, Jay $\mathrm{Wu}$,Tzong-Jer Chen, Fuzzy c-means clustering with spatial information for image segmentation, September,6, 2005 .

[2] Z. M. Wang, Y. C. Soh, Q. Song, and K. Sim, "Adaptive spatial information-theoretic clustering for image segmentation,", vol. 42, no. 9, pp. 2029-2044, 2009

[3] M. Kass, A. Witkin, and D.Terzopoulos, "Snakes: Active contour models," Int. J. Comput. Vis., vol.1, pp. 321331,1987 .
[4] S. Osher and J. Sethian, "Fronts propagating with curvature dependent speed: Algorithms based on Hamilton-Jacobi formulations," J. Comp. Phys., vol. 79, pp. 12-49, 1988.

[5] Tsai, Y.-H.R., Cheng, L.-T., Osher, S., Zhao, H.-K.: Fast sweeping algorithms for a class of Hamilton-Jacobi equations. SIAM J. Numer. Anal. 41, 673-694 (2003).

[6] S. Osher and R.P. Fedkiw. Level set methods and dynamic implicit surfaces. Springer, 2003.

[7] Chunming Li , Chenyang Xu , Changfeng Gui , and Martin D. Fox , "Level Set Evolution Without Reinitialization: A New Variational Formulation", Proceedings of the 2005 IEEE Computer Society Conference on Computer Vision and Pattern Recognition (CVPR'05) 1063-6919/05 \$20.00 @ 2005 IEEE.

[8] Yang MS, Hu YJ, Lin KCR, Lin CCL. Segmentation techniques fortissue differentiation in MRI of Ophthalmology using fuzzy clustering algorithms. Magn Reson Imaging 2002;20:173-9.

[9] J. A. Sethian, Level set methods and fast marching methods, Cambridge: Cambridge University Press, 1999.

[10] B. Vemuri and Y. Chen, "Joint image registration and segmentation", Geometric Level Set Methods in Imaging, Vision, and Graphics, Springer, pp. 251-269, 2003. 